\title{
Cardiovascular adverse events associated with oral antineoplastic therapy
}

\author{
Eventos adversos cardiovasculares associados à terapia antineoplásica oral \\ Eventos adversos cardiovasculares asociados a la terapia antineoplásica oral
Jacqueline Mota da Silva', Beatriz da Silva Lima', Thelma Leite de Araújo', Francisca Elisângela Teixeira Lima', Gilmara Holanda da Cunha'

' Universidade Federal do Ceará, Faculty of Pharmacy, Dentistry and Nursing, Department of Nursing. Fortaleza, Ceará, Brazil.

How to cite this article:

Silva JM, Lima BS, Araújo TL, Lima FET, Cunha GH. Cardiovascular adverse events associated with oral antineoplastic therapy. Rev Bras Enferm [Internet]. 2018;71(5):2561-9. DOI: http://dx.doi.org/10.1590/0034-7167-2017-0450

\author{
Submission: 06-19-2017 Approval: 10-18-2017
}

\section{ABSTRACT}

Objective: To identify in the literature the cardiovascular adverse events resulting from oral antineoplastic therapy. Method: Integrative review of the literature through the SCOPUS, Scientific Electronic Library Online (SciELO), Cumulative Index to Nursing and Allied Health Literature (CINAHL), Medical Literature Analysis and Retrieval System Online (MEDLINE) databases. The antineoplastic, cardiotoxicity, cardiovascular system and adverse reaction descriptors were used in Portuguese, English and Spanish. We selected 23 articles published between 1985 and 2015. Results: Twenty studies were related to cardiac events and eleven to peripheral vascular events. The most frequent adverse cardiac events were reduced left ventricular ejection fraction, myocardial infarction, changes in the electrocardiogram, heart failure and angina, whereas peripheral vascular events were hypertension and thromboembolism. Conclusion: Oral antineoplastic therapy is associated with different adverse events, including cardiac and peripheral vascular events. Descriptors: Antineoplastic Agents; Adverse Effects; Cardiotoxicity; Cardiovascular System; Review.

\section{RESUMO}

Objetivo: Identificar na literatura os eventos adversos cardiovasculares decorrentes da terapia antineoplásica oral. Método: Revisão integrativa de literatura nas bases de dados SCOPUS, Scientific Electronic Library Online (SciELO), Cumulative Index to Nursing and Allied Health Literature (CINAHL) e Medical Literature Analysis and Retrieval System Online (MEDLINE). Utilizaram-se os descritores antineoplásicos, cardiotoxicidade, sistema cardiovascular e reação adversa, em português, inglês e espanhol. Foram selecionados 23 artigos publicados entre 1985 e 2015. Resultados: Vinte estudos estavam relacionados aos eventos cardíacos e onze aos eventos vasculares periféricos. Os eventos adversos cardíacos mais frequentes foram diminuição da fração de ejeção do ventrículo esquerdo, infarto do miocárdio, alterações no eletrocardiograma, insuficiência cardíaca e angina, enquanto que os vasculares periféricos foram hipertensão arterial e tromboembolismo. Conclusão: A terapia antineoplásica oral está associada a diferentes eventos adversos, dentre eles os cardíacos e os vasculares periféricos.

Descritores: Antineoplásicos; Efeitos Colaterais e Reações Adversas Relacionados a Medicamentos; Cardiotoxicidade; Sistema Cardiovascular; Revisão.

\section{RESUMEN}

Objetivo: Identificar en la literatura los eventos adversos cardiovasculares derivados de la terapia antineoplásica oral. Método: Revisión integrativa de literatura en las bases de datos SCOPUS, Scientific Electronic Library Online (SciELO), Cumulative Index to Nursing and Allied Health Literature (CINAHL) y Medical Literature Analysis and Retrieval System Online (MEDLINE). Se utilizaron descriptores antineoplásicos, cardiotoxicidad, el sistema cardiovascular y reacción adversa en Portugués, Inglés y Español. Se seleccionaron 23 artículos publicados entre 1985 y 2015. Resultados: Veinte estudios estaban relacionados con los eventos cardiacos y once con los eventos vasculares periféricos. Los eventos adversos cardiacos más frecuentes fueron la disminución de la fracción de eyección del ventrículo izquierdo, infarto de miocardio, cambios en el electrocardiograma, insuficiencia cardíaca y angina, mientras que los vasculares periféricos fueron hipertensión arterial y tromboembolismo. Conclusión: La terapia antineoplásica oral está asociada a diferentes eventos adversos, entre ellos los cardíacos y los vasculares periféricos.

Descriptores: Antineoplásicos; Efectos Colaterales y Reacciones Adversas Relacionados con Medicamentos; Cardiotoxicidad; Sistema Cardiovascular; Revisión. 


\section{INTRODUCTION}

Neoplasms are among the leading causes of morbidity and mortality in the world and represent challenges to global health, being curable since treated early and properly. Effective treatment requires careful selection of the intervention, so that the chances of cure are increased. Among the existing modalities, oral antineoplastic therapy is a frequent choice, which may or may not be associated with other treatments, such as surgery, radiotherapy and hormone therapy ${ }^{(1)}$.

The use of oral antineoplastic therapy requires responsibility on the part of the patient, as well as the health team. Its use offers some advantages, like the decrease of discomfort, since it does not need venous access; use of medication at home or at work, without compromising the usual routine; and its simple and quick administration. However, this new reality requires specific guidance and educational practices by the health team to occur appropriate therapy and recognize adverse events ${ }^{(2)}$. These changes are signs, symptoms, sickness or laboratory findings that are not favorable, which are temporary or not, associated with the treatment ${ }^{(3)}$.

Cardiovascular adverse events are highlighted as representing a great risk to the patient, since the cardiotoxicity of the drugs can lead to serious damage and death ${ }^{(4)}$. These adverse events give rise to resolutive actions by the nurse and other members of the health team who must be supported by scientific knowledge in order to recognize adversities and intervene promptly in harm reduction, as well as to guide and encourage the patient to participate in the treatment decisions ${ }^{(5-6)}$.

The scientific literature has detailed the events associated with antineoplastic agents in general ${ }^{(7)}$, but in relation to oral therapy, there is still a need to synthesize and analyze the existing studies. Thus, the following research question was formulated: what are the adverse cardiovascular events resulting from oral antineoplastic therapy, according to the scientific literature on health?

\section{OBJECTIVE}

We aim to identify in the literature cardiovascular adverse events resulting from oral antineoplastic therapy.

\section{METHOD}

In order to reach the proposed objective, an integrative review of literature was carried out. For its development, six stages were covered: identification of the theme and formulation of the research question; sampling; categorization of studies; synthesis of knowledge; discussion and interpretation of results; and evaluation of the studies included in the review ${ }^{(8)}$.

This study has as its core theme the cardiovascular health. In order to formulate the research question, the PICO strategy was used, which includes the fundamental elements for question construction and bibliographic search for evidence. PICO stands for the acronym of $\mathrm{P}=$ Patient; $\mathrm{I}=$ Intervention; $\mathrm{C}=$ Control and $\mathrm{O}=$ Outcomes $^{(9)}$. In this study, the elements presented are: Patient: people with cancer; intervention: administration of oral antineoplastic agents; cardiovascular adverse events. In view of its objective, being a research and an integrative review, the comparison stage $(\mathrm{C})$ was not performed.

We investigated the publications in the SCOPUS, Scientific Electronic Library Online (SciELO), Cumulative Index to Nursing and Allied Health Literature (CINAHL), and Medical Literature Analysis and Retrieval System Online (MEDLINE) databases. We used the Health Science Descriptors (DeCS) of the Virtual Health Library (VHL) and the Medical Subject Headings (MeSH) of the National Library of Medicine to search (antineoplastic agents, cardiotoxicity, cardiovascular system and adverse reaction descriptors in the Portuguese, English and Spanish languages depending on the search base. At each base the descriptors were combined with the Boolean operator AND in two ways: antineoplastic agents AND cardiotoxicity AND adverse reaction; and antineoplastic agents AND cardiovascular system AND adverse reaction. The search for studies in the literature was carried out by four researchers, who worked simultaneously and separately on all bases, with the aid of a research protocol containing the following topics: title of the article; authors of the article; title of the journal; study design; medication used; population of the study; and identified adverse events. The process of searching and selecting articles occurred between May and July 2016.

Studies describing adverse cardiovascular events from treatment with oral antineoplastic agents were selected. As criteria for inclusion, were established surveys that responded to the guiding question, published in the Portuguese, English or Spanish languages, available in full through electronic means, regardless of the year of publication and that belonged to I, II, III and IV levels of evidence. Studies addressing combinations of oral antineoplastic agent and other therapies (intravenous, intrathecal, radiotherapy), review articles and repeated ones, experiments on individuals without cancer, in vitro and animal studies took part in the exclusion criteria.

The classification was established according to level of evidence. Level I: Metanalysis of randomized clinical trials; Level II: Randomized clinical trials; Level III: Clinical trials without randomization; and Level IV: well-delineated casecontrol and cohort studies ${ }^{(10)}$. After searching the databases, the selected articles were analyzed in their full. Figure 1 shows the searched data.

The selection and analysis of the selected studies were carried out simultaneously by two nursing researchers and students of the postgraduate program in Nursing working together and a consensus meeting was held on the studies that composed the sample. For this, the articles were organized in the database through the synthesis of the following information: samples, objectives, methods employed, results and conclusions.

In addition, the researchers discussed the convergences and divergences between the results and conclusions of the studies, analyzing the relationships of the articles with the guiding question of the review. At this stage, we also sought to identify gaps in knowledge about the subject matter. Finally, the results were formulated from the synthesis of knowledge found in the selected studies, which was divided into two categories: 1. Cardiac adverse events; and 2. Peripheral vascular adverse events. 


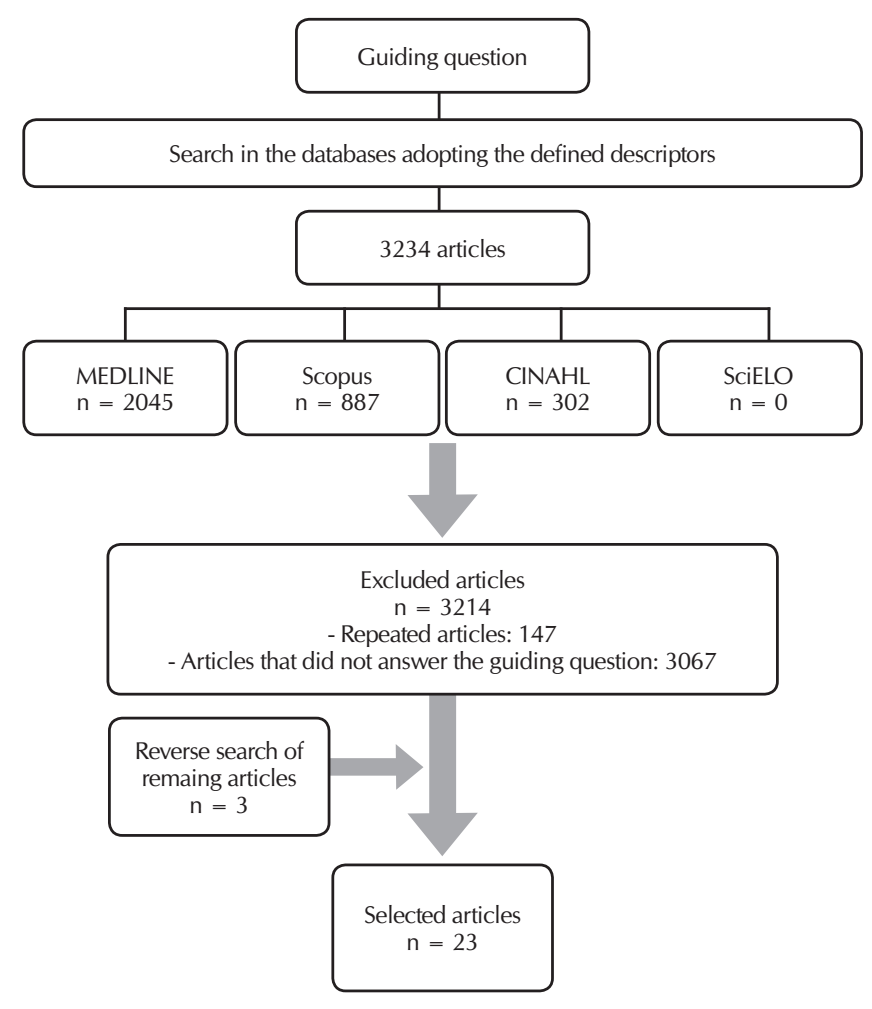

Figure 1 - Distribution of articles found and selected, Fortaleza, Ceará State, Brazil, 2016

\section{RESULTS}

The characterization of the 23 articles showed that the year of publication ranged between 1985 and 2015, with thirteen published in the United States ${ }^{(11-23)}$, seven in England ${ }^{(24-30)}$ and three in other European countries ${ }^{(31-33)}$. Regarding the levels of evidence, the following distribution was observed: one level $\mathrm{I}^{(27)}$, seven level II ${ }^{(15,17,20,22,28-29,31)}$, six level III ${ }^{(13,18-19,21,23,30)}$, and nine level IV ${ }^{(11-12,14,16,24,26,32-33)}$.

Considering the cardiac adverse events and peripheral vascular adverse events, most of the articles were part of the first with twenty studies. In it, the reduced left ventricular ejection fraction $^{(12,14-15,17,20-21,24-26,30)}$, myocardial infarction ${ }^{(14,25,29,32)}$, alterations on the electrocardiogram ${ }^{(12-13,16,19,27,30-32)}$, heart failure ${ }^{(11,16,25-26)}$, and angina ${ }^{(22,29,32)}$.

The second category of articles involved peripheral vascular adverse events related to oral antineoplastic therapy and consisted of eleven articles highlighting the outcomes, hyperten$\operatorname{sion}^{(11,13,15,25-26,28-29,31)}$ and thromboembolic events ${ }^{(12,23,29)}$.

15,941 patients participated in the 23 studies evaluated. The most commonly used oral antineoplastic agents were tyrosine kinase inhibitors, with sixteen studies $(69.5 \%)$, and studies on sunitinib $^{(11-15,24-28)}$, pazopanib ${ }^{(11,27,31)}$, sorafenib ${ }^{(11-12,27)}$, imatinib $^{(16)}$, lapatinib $^{(17)}$, nilotinib ${ }^{(32)}$, telatinib ${ }^{(18)}$, dasatinib(19) ${ }^{(19)}$ axitinib, vandetanib, cabozantinib, ponatinib and regorafenib ${ }^{(27)}$. The most frequent types of cancer were renal cell carcinoma ${ }^{(11-12,14-15,24,26)}$ followed by breast cancer ${ }^{(17,20,29-30)}$.

Chart 1 - Cardiac adverse events associated with oral antineoplastic therapy

\begin{tabular}{|c|c|c|c|}
\hline Title & $\begin{array}{l}\text { Country/ } \\
\text { Year }\end{array}$ & $\begin{array}{l}\text { Design/ } \\
\text { Study sample }\end{array}$ & Outcome \\
\hline $\begin{array}{l}\text { The frequency and severity of } \\
\text { cardiovascular toxicity from } \\
\text { targeted therapy in advanced renal } \\
\text { cell carcinoma patients }{ }^{(11)}\end{array}$ & $\begin{array}{c}\text { United } \\
\text { States/ } \\
2013\end{array}$ & $\begin{array}{l}\text { Cohort } \\
289 \text { patients with } \\
\text { advanced renal cell } \\
\text { carcinoma }\end{array}$ & $\begin{array}{l}\text { - Sunitinib: heart failure }(2 \%) \text {, coronary artery disease } \\
(9 \%) \text { and atrial fibrillation }(1 \%) \text {. } \\
\text { - Sorafenib: heart failure }(4 \%) \text {, coronary artery disease } \\
(5 \%) \text { and atrial fibrillation }(4 \%) \text {. } \\
\text { - Pazopanib: heart failure }(2 \%) \text {, coronary artery disease } \\
(2 \%) \text { and atrial fibrillation }(5 \%) \text {. }\end{array}$ \\
\hline $\begin{array}{l}\text { Cardiac toxicity of sunitinib } \\
\text { and sorafenib in patients with } \\
\text { metastatic renal cell carcinoma }\end{array}$ & $\begin{array}{l}\text { United } \\
\text { States, } \\
2008\end{array}$ & $\begin{array}{l}\text { Cohort } \\
86 \text { patients with metastatic } \\
\text { renal cell carcinoma }\end{array}$ & $\begin{array}{l}\text { - Sunitinib or sorafenib: changes in electrocardiogram } \\
(46.5 \%) \text {, reduced left ventricular ejection fraction } \\
(10.4 \%) \text {; pericardial effusion }(1 \%) \text {, rise in cardiac } \\
\text { enzymes }(30 \%) \text {. }\end{array}$ \\
\hline $\begin{array}{l}\text { Phase I and Pharmacokinetic Study } \\
\text { of Sunitinib in Pediatric Patients } \\
\text { with Refractory Solid Tumors: } \\
\text { A Children's Oncology Group } \\
\text { Study }{ }^{(13)}\end{array}$ & $\begin{array}{l}\text { United } \\
\text { States, } \\
2010\end{array}$ & $\begin{array}{l}\text { Clinical trial } \\
33 \text { patients from } 2 \text { to } 21 \\
\text { years old with refractory } \\
\text { solid tumors }\end{array}$ & $\begin{array}{l}\text { - Sunitinib: Left ventricular systolic dysfunction }(8.6 \%) \text {, } \\
\text { prolongation of QTc on the electrocardiogram }(8.6 \%) \text {. }\end{array}$ \\
\hline $\begin{array}{l}\text { Hyperlipidemia and } \\
\text { Hypothyroidism Among Metastatic } \\
\text { Renal Cell Carcinoma Patients } \\
\text { Taking Sunitinib Malate }{ }^{(14)}\end{array}$ & $\begin{array}{c}\text { United } \\
\text { States, } \\
2015\end{array}$ & $\begin{array}{l}\text { Cohort } \\
39 \text { patients with metastatic } \\
\text { renal cell carcinoma }\end{array}$ & $\begin{array}{l}\text { - Sunitinib: decrease of left ventricular function and } \\
\text { death after myocardial infarction }(2.5 \%) \text {. }\end{array}$ \\
\hline $\begin{array}{l}\text { Sunitinib versus Interferon Alfa in } \\
\text { Metastatic Renal-Cell Carcinoma }{ }^{(15)}\end{array}$ & $\begin{array}{l}\text { United } \\
\text { States, } \\
2007\end{array}$ & $\begin{array}{l}\text { Clinical trial } \\
750 \text { patients with } \\
\text { metastatic renal cell } \\
\text { carcinoma }\end{array}$ & $\begin{array}{l}\text { - Sunitinib: reduced left ventricular ejection fraction }(2 \%) \text {. } \\
\text { - Interferon alfa: decrease of left ventricular ejection fraction } \\
(1 \%) \text {. }\end{array}$ \\
\hline $\begin{array}{l}\text { Rare Incidence of Congestive Heart } \\
\text { Failure in Gastrointestinal Stromal } \\
\text { Tumor and Other Sarcoma Patients } \\
\text { Receiving Imatinib Mesylate }(I M)^{(16)}\end{array}$ & $\begin{array}{l}\text { United } \\
\text { States, } \\
2010\end{array}$ & $\begin{array}{c}\text { Cohort } \\
219 \text { patients with sarcoma }\end{array}$ & $\begin{array}{l}\text { - Imatinibe: myocardial ischemia or chest pain }(2.3 \%) \text {, } \\
\text { arrhythmias }(0.9 \%) \text {; alterations in the electrocardiogram } \\
(1 \%) \text {, left ventricular dysfunction }(0.4 \%) \text {, cardiac arrest } \\
(0.4 \%) \text {, heart failure }(0.5 \%) \text {. }\end{array}$ \\
\hline
\end{tabular}


Chart 1 (concluded)

\begin{tabular}{|c|c|c|c|}
\hline Title & $\begin{array}{l}\text { Country/ } \\
\text { Year }\end{array}$ & $\begin{array}{l}\text { Design/ } \\
\text { Study sample }\end{array}$ & Outcome \\
\hline $\begin{array}{l}\text { Randomized study of lapatinib } \\
\text { alone or in combination with } \\
\text { trastuzumab in women with ErbB2- } \\
\text { positive, trastuzumab-refractory } \\
\text { metastatic breast cancer }{ }^{(17)}\end{array}$ & $\begin{array}{l}\text { United } \\
\text { States, } \\
2010\end{array}$ & $\begin{array}{c}\text { Clinical trial } \\
296 \text { women with ErbB2- } \\
\text { positive metastatic breast } \\
\text { cancer }\end{array}$ & $\begin{array}{l}\text { - Lapatinib: reduced left ventricular ejection fraction } \\
(2 \%) \text {. }\end{array}$ \\
\hline $\begin{array}{l}\text { Phase } 1 \text { Pharmacokinetic and } \\
\text { Drug-Interaction Study of Dasatinib } \\
\text { in Patients With Advanced Solid } \\
\text { Tumors }{ }^{(19)}\end{array}$ & $\begin{array}{l}\text { United } \\
\text { States, } \\
2010\end{array}$ & $\begin{array}{l}\text { Clinical trial } \\
29 \text { patients with advanced } \\
\text { solid tumors }\end{array}$ & $\begin{array}{l}\text { - Dasatinib: QTC interval prolongation on the } \\
\text { electrocardiogram }(3.5 \%) \text {. }\end{array}$ \\
\hline $\begin{array}{l}\text { A Prospective Randomized Trial of } \\
\text { Doxorubicin Versus Idarubicin in } \\
\text { the Treatment of Advanced Breast } \\
\text { Cancer }{ }^{(20)}\end{array}$ & $\begin{array}{l}\text { United } \\
\text { States, } \\
1989\end{array}$ & $\begin{array}{c}\text { Clinical trial } \\
76 \text { patients with } \\
\text { advanced breast cancer }\end{array}$ & $\begin{array}{l}\text { - Idarubicin: reduced left ventricular ejection fraction } \\
(5.8 \%)\end{array}$ \\
\hline $\begin{array}{l}\text { Phase I Clinical Trial } \\
\text { of Orally Administered } \\
\text { 4-Demethoxydaunorubicin } \\
\text { (Idarubicin) with Pharmacokinetic } \\
\text { and in Vitro Drug Sensitivity } \\
\text { Testing in Children with Refractory } \\
\text { Leukemia }^{(21)}\end{array}$ & $\begin{array}{l}\text { United } \\
\text { States, } \\
1988\end{array}$ & $\begin{array}{l}\text { Clinical trial } \\
15 \text { children with acute } \\
\text { refractory leukemia }\end{array}$ & $\begin{array}{l}\text { - Idarubicin: reduced left ventricular ejection fraction } \\
(6.6 \%) \text { and of fractional shortening }(40 \%) \text {. }\end{array}$ \\
\hline $\begin{array}{l}\text { Capecitabine, an Oral } \\
\text { Fluoropyrimidine Carbamate With } \\
\text { Substantial Activity in Advanced } \\
\text { Colorectal Cancer: Results of a } \\
\text { Randomized Phase II Study (22) }\end{array}$ & $\begin{array}{l}\text { United } \\
\text { States, } \\
2000\end{array}$ & $\begin{array}{c}\text { Clinical trial } \\
109 \text { patients with } \\
\text { advanced colorectal } \\
\text { cancer }\end{array}$ & $\begin{array}{l}\text { - Capecitabine: Angina }(1.8 \%) \text {, atrial fibrillation }(0.9 \%) \\
\text { and chest pain }(2.7 \%) \text {. }\end{array}$ \\
\hline $\begin{array}{l}\text { Cardiotoxicity associated with the } \\
\text { cancer therapeutic agent sunitinib }_{\text {malate }^{(24)}}\end{array}$ & $\begin{array}{l}\text { England, } \\
2008\end{array}$ & $\begin{array}{l}\text { Cohort } \\
48 \text { patients with renal cell } \\
\text { cancer or gastrointestinal } \\
\text { stromal tumor }\end{array}$ & $\begin{array}{l}\text { - Sunitinib: ventricular dysfunction with the reduced left } \\
\text { ventricular ejection fraction }(12.5 \%) \text { and cardiogenic } \\
\text { shock }(2 \%) \text {. }\end{array}$ \\
\hline $\begin{array}{l}\text { Cardiotoxicity Associated with } \\
\text { the Tyrosine Kinase Inhibitor } \\
\text { Sunitinib(25) }\end{array}$ & $\begin{array}{l}\text { England, } \\
2007\end{array}$ & $\begin{array}{l}\text { Cohort } \\
75 \text { patients with imatinib } \\
\text { resistant gastrointestinal } \\
\text { stromal tumor }\end{array}$ & $\begin{array}{l}\text { - Sunitinib: congestive heart failure }(8 \%) \text {, reduced left } \\
\text { ventricular ejection fraction }(28 \%) \text {, rise in troponin }(18 \%) \\
\text { and death after myocardial infarction }(1.3 \%) \text {. }\end{array}$ \\
\hline $\begin{array}{l}\text { Cardiovascular toxicity following } \\
\text { sunitinib therapy in metastatic } \\
\text { renal cell carcinoma: a multicenter } \\
\text { analysis }{ }^{(26)}\end{array}$ & $\begin{array}{l}\text { England, } \\
2009\end{array}$ & $\begin{array}{l}\text { Cohort } \\
175 \text { patients with } \\
\text { metastatic renal cell } \\
\text { carcinoma }\end{array}$ & $\begin{array}{l}\text { - Sunitinib: reduced left ventricular ejection fraction } \\
(18.9 \%) \text { and congestive heart failure }(6.9 \%) \text {. }\end{array}$ \\
\hline $\begin{array}{l}\text { QTC interval prolongation with } \\
\text { vascular endothelial growth } \\
\text { factor receptor tyrosine kinase } \\
\text { inhibitors }\end{array}$ & $\begin{array}{l}\text { England, } \\
2015\end{array}$ & $\begin{array}{l}\text { Metanalysis } \\
6548 \text { patients with } \\
\text { hepatocellular carcinoma, } \\
\text { soft tissue sarcoma, renal } \\
\text { cell carcinoma, colorectal, } \\
\text { prostate, pancreatic, } \\
\text { breast, thyroid, lung or } \\
\text { gallbladder cancer }\end{array}$ & $\begin{array}{l}\text { - Tyrosine kinase inhibitors in general: changes in } \\
\text { electrocardiogram }(4.4 \%, \mathrm{RR}=66, \mathrm{Cl}=95 \% \text { and } \mathrm{p}=0.001 \\
\text { in relation to other antineoplastic agents). } \\
\text { - Axitinib: ventricular tachycardia }(0.33 \%) \text {. } \\
\text { - Capecitabine: Atrial fibrillation }(0.93 \%) \text {. } \\
\text { - Vandetanib: Atrial fibrillation }(0.56 \%) \text {, ventricular } \\
\text { tachycardia ( } 1.37 \%) \text { and cardiac arrest }(0.15 \%) \text {. } \\
\text { - Pazopanib: atrial fibrillation }(0.37 \%) \text { and cardiac arrest } \\
(0.34 \%) \text {. }\end{array}$ \\
\hline $\begin{array}{l}\text { Comprehensive side-eff ect profi } \\
\text { le of anastrozole and tamoxifen as } \\
\text { adjuvant treatment for early-stage } \\
\text { breast cancer: long-term safety } \\
\text { analysis of the ATAC trial(29) }\end{array}$ & $\begin{array}{l}\text { England, } \\
2006\end{array}$ & $\begin{array}{l}\text { Clinical trial } \\
6241 \text { women after } \\
\text { menopause with breast } \\
\text { cancer }\end{array}$ & $\begin{array}{l}\text { - Anastrozole: angina, myocardial infarction, myocardial } \\
\text { ischemia and coronary artery disease }(2 \%) \text {. } \\
\text { - Tamoxifeno: Angina, myocardial ischemia and coronary } \\
\text { artery disease }(2 \%) \text { and myocardial infarction }(1 \%) \text {. }\end{array}$ \\
\hline $\begin{array}{l}\text { Activity of } 4 \\
\text { Demethoxydaunorubicin by the } \\
\text { Oral Route in Advanced Breast } \\
\text { Cancer }\end{array}$ & $\begin{array}{l}\text { England, } \\
1985\end{array}$ & $\begin{array}{l}\text { Clinical trial } \\
29 \text { patients with advanced } \\
\text { breast cancer }\end{array}$ & $\begin{array}{l}\text { - Idarrubicina: Changes in electrocardiogram }(6.8 \%) \text { and } \\
\text { reduced left ventricular ejection fraction }(3.4 \%) \text {. }\end{array}$ \\
\hline $\begin{array}{l}\text { A randomized, double-blind, } \\
\text { placebo-controlled study to evaluate } \\
\text { the effect of repeated oral doses of } \\
\text { pazopanib on cardiac conduction } \\
\text { in patients with solid tumors }\end{array}$ & $\begin{array}{l}\text { Germany, } \\
2013\end{array}$ & $\begin{array}{l}\text { Clinical trial } \\
95 \text { patients with advanced } \\
\text { solid tumors }\end{array}$ & $\begin{array}{l}\text { - Pazopanib: decreased heart rate }(\text { mean }=9.9 \mathrm{bpm}) \text {, } \\
\text { prolongation of } 4.4 \mathrm{~ms} \text { in the QTc interval }(90 \% \mathrm{Cl} \text { : } \\
-2.4,11.2), \text { compared to placebo. }\end{array}$ \\
\hline
\end{tabular}


Chart 1 (concluded)

\begin{tabular}{|c|c|c|c|}
\hline Title & $\begin{array}{l}\text { Country/ } \\
\text { Year }\end{array}$ & $\begin{array}{l}\text { Design/ } \\
\text { Study sample }\end{array}$ & Outcome \\
\hline $\begin{array}{l}\text { Clinical cardiac safety profile of } \\
\text { nilotinib }{ }^{(32)}\end{array}$ & $\begin{array}{l}\text { Italy, } \\
2012\end{array}$ & $\begin{array}{l}\text { Cohort } \\
81 \text { patients previously } \\
\text { treated with nilotinib }\end{array}$ & $\begin{array}{l}\text { - Nilotinib: Myocardial infarction }(2.4 \%) \text {, acute coronary } \\
\text { syndrome }(7.4 \%) \text {, atrial fibrillation }(1.2 \%) \text {, angina }(1.2 \%) \\
\text { and changes in electrocardiogram }(20 \%) \text {. }\end{array}$ \\
\hline $\begin{array}{l}\text { Increased prevalence of subclinical } \\
\text { cardiac valve fibrosis in patients } \\
\text { with prolactinomas on long-term } \\
\text { bromocriptine and cabergoline }_{\text {treatment }^{(33)}}\end{array}$ & $\begin{array}{l}\text { Norway, } \\
2012\end{array}$ & $\begin{array}{l}\text { Case-control } \\
334 \text { patients with } \\
\text { prolactinoma }\end{array}$ & $\begin{array}{l}\text { - Cabergoline: Asymptomatic pericardial effusion }(0.9 \%) \\
\text { and risk of cardiac fibrosis }(\mathrm{OR}=2.27, \mathrm{p}=0.016) . \\
\text { - Bromocriptine: Asymptomatic pericardial effusion } \\
(1.8 \%) \text { and risk of cardiac fibrosis }(\mathrm{OR}=2.66, \mathrm{p}=0.014) \text {. }\end{array}$ \\
\hline
\end{tabular}

Chart 2 - Peripheral vascular adverse events associated with oral antineoplastic therapy

\begin{tabular}{|c|c|c|c|}
\hline Title & $\begin{array}{l}\text { Year/ } \\
\text { Country }\end{array}$ & $\begin{array}{c}\text { Design/ } \\
\text { Study sample }\end{array}$ & Outcome \\
\hline $\begin{array}{l}\text { The frequency and severity of } \\
\text { cardiovascular toxicity from targeted } \\
\text { therapy in advanced renal cell } \\
\text { carcinoma patients }^{(11)}\end{array}$ & $\begin{array}{c}\text { United States, } \\
2013\end{array}$ & $\begin{array}{l}\text { Cohort } \\
289 \text { patients with } \\
\text { advanced renal cell } \\
\text { carcinoma }\end{array}$ & $\begin{array}{l}\text { - Sunutinib: hypertension }(44 \%) \text {. } \\
\text { - Sorafenib: hypertension }(52 \%) \text {. } \\
\text { - Pazopanib: hypertension }(49 \%) \text {. }\end{array}$ \\
\hline $\begin{array}{l}\text { Cardiac Toxicity of Sunitinib and } \\
\text { Sorafenib in Patients With Metastatic } \\
\text { Renal Cell Carcinoma }\end{array}$ & $\begin{array}{l}\text { United States, } \\
2008\end{array}$ & $\begin{array}{l}\text { Cohort } \\
86 \text { patients with metstatic } \\
\text { renal cell carcinoma }\end{array}$ & $\begin{array}{l}\text { - Sunitinib or sorafenib: ischemic optic } \\
\text { neuropathy and occlusion of the posterior } \\
\text { tibial artery }(1 \%) \text {. }\end{array}$ \\
\hline $\begin{array}{l}\text { Phase I and Pharmacokinetic Study } \\
\text { of Sunitinib in Pediatric Patients with } \\
\text { Refractory Solid Tumors: A Children's } \\
\text { Oncology Group Study }{ }^{(13)}\end{array}$ & $\begin{array}{c}\text { United States, } \\
2010\end{array}$ & $\begin{array}{c}\text { Clinical trial } \\
33 \text { patients de } 2 \text { a } 21 \\
\text { anos, com refractory solid } \\
\text { tumors }\end{array}$ & - Sunitinib: hypertension (12\%). \\
\hline $\begin{array}{l}\text { Sunitinib versus Interferon Alfa in } \\
\text { Metastatic Renal-Cell Carcinoma }{ }^{(15)}\end{array}$ & $\begin{array}{c}\text { United States, } \\
2007\end{array}$ & $\begin{array}{c}\text { Clinical trial } \\
750 \text { patients with } \\
\text { carcinoma de célula renal } \\
\text { metastático }\end{array}$ & - Sunitinib: hypertension (8\%). \\
\hline $\begin{array}{l}\text { Hypertension and Rarefaction during } \\
\text { Treatment with Telatinib, a Small } \\
\text { Molecule Angiogenesis Inhibitor }{ }^{(18)}\end{array}$ & $\begin{array}{l}\text { United States, } \\
\quad 2008\end{array}$ & $\begin{array}{l}\text { Clinical trial } \\
18 \text { patients with solid } \\
\text { tumors }\end{array}$ & $\begin{array}{l}\text { - Telatinib: rise in blood pressure }(78 \%) \\
\text { (aortic pulse wave velocity increased in } 94 \% \\
\text { of patients, } p=0.001) \text {, decreased skeletal } \\
\text { muscle capillary density and decreased blood } \\
\text { flow in the skin }(p=0.015) \text {. }\end{array}$ \\
\hline $\begin{array}{l}\text { Oral Melphalan, Prednisone, and } \\
\text { Thalidomide for Newly Diagnosed } \\
\text { Patients with Myeloma }\end{array}$ & $\begin{array}{l}\text { United States, } \\
\quad 2005\end{array}$ & $\begin{array}{c}\text { Clinical trial } \\
41 \text { patients with multiple } \\
\text { myeloma }\end{array}$ & $\begin{array}{l}\text { - Melphalan }+ \text { Prednisone }+ \text { thalidomide: } \\
\text { thromboembolic Events }(20 \%)\end{array}$ \\
\hline $\begin{array}{l}\text { Cardiovascular toxicity Associated } \\
\text { with the Tyrosine Kinase Inhibitor } \\
\text { Sunitinib(25) }\end{array}$ & England, 2007 & $\begin{array}{l}\text { Cohort } \\
75 \text { patients with imatinib } \\
\text { resistant gastrointestinal } \\
\text { stromal tumor }\end{array}$ & - Sunitinib: hypertension (47\%). \\
\hline $\begin{array}{l}\text { Cardiovascular toxicity following } \\
\text { sunitinib therapy in metastatic } \\
\text { renal cell carcinoma: a multicenter } \\
\text { analysis }^{(26)}\end{array}$ & England, 2009 & $\begin{array}{l}\text { Cohort } \\
175 \text { patients with } \\
\text { metastatic cell carcinoma }\end{array}$ & -Sunitinib: hypertension $(13.7 \%)$. \\
\hline $\begin{array}{l}\text { Efficacy and safety of sunitinib } \\
\text { in patients with advanced } \\
\text { gastrointestinal stromal tumour after } \\
\text { failure of imatinib: a randomised } \\
\text { controlled trial(28) }\end{array}$ & England, 2006 & $\begin{array}{l}\text { Clinical trial } \\
312 \text { patients with imatinib } \\
\text { resistant gastrointestinal } \\
\text { stromal tumor }\end{array}$ & - Sunitinib: hypertension (11\%). \\
\hline $\begin{array}{l}\text { Comprehensive side-eff ect profi } \\
\text { le of anastrozole and tamoxifen as } \\
\text { adjuvant treatment for early-stage } \\
\text { breast cancer: long-term safety } \\
\text { analysis of the ATAC trial(29) }\end{array}$ & England, 2006 & $\begin{array}{c}\text { Clinical trial } \\
6241 \text { women after } \\
\text { menopause with breast } \\
\text { cancer }\end{array}$ & $\begin{array}{l}\text { - Anastrozole: venous thromboembolic events } \\
(3 \%) \text { and hypertension }(13 \%) \text {. } \\
\text { - Tamoxifen: thromboembolic venous events } \\
\text { and hypertension }(11 \%) \text {. }\end{array}$ \\
\hline $\begin{array}{l}\text { A randomized, double-blind, } \\
\text { placebo-controlled study to evaluate } \\
\text { the effect of repeated oral doses of } \\
\text { pazopanib on cardiac conduction in } \\
\text { patients with solid tumors }{ }^{(31)}\end{array}$ & Germany, 2013 & $\begin{array}{l}\text { Clinical trial } \\
95 \text { patients with advanced } \\
\text { solid tumors }\end{array}$ & $\begin{array}{l}\text { - Pazopanib: Transient increase in sys- } \\
\text { tolic blood pressure (mean }+17.7 \mathrm{mmHg}, \\
\mathrm{SD}=14.2) \text { and diastolic }(\text { mean }+11.9 \mathrm{mmHg} \text {, } \\
\mathrm{SD}=6.3) \text { and hypertension }(15 \%) .\end{array}$ \\
\hline
\end{tabular}




\section{DISCUSSION}

The research about cardiovascular adverse events resulting from the use of oral antineoplastic agents has allowed the identification of risk situations in which the patient undergoing cancer treatment is submitted ${ }^{(34)}$. Several peripheral vascular and cardiac adverse events were observed.

Adverse events occurring during antineoplastic therapy are mainly due to drug toxicity. In view of this toxicity, these events are classified as levels 0 and 4,0 representing an unchecked event and 4 representing greater severity with risk of death or incapacitating impairment ${ }^{(35)}$. In the analyzed studies, the verified events were classified from grade 1 to 4 , evidencing from transient alterations and without major complications, to those that represent great risk for the patient, including the occurrence of deaths.

Cardiac adverse events were: myocardial infarction, changes in electrocardiogram, heart failure, angina, and, most commonly, reduced left ventricular ejection fraction, with tyrosine kinase inhibitors being the most involved drugs. Among this drug class, treatment with sunitinib followed by reduced ventricular ejection fraction was reported in five studies, with incidence ranging from $2 \%$ to $28 \%^{(12-13,24-26)}$. The literature shows that such changes occur in a large part of the patients, so monitoring and monitoring is necessary until the conclusion of the therapy, in order to prevent and diagnose such events early, as well as to treat them correctly ${ }^{(25,36)}$. One of the determining factors for the prevention of adverse events is the administration of the appropriate dose of the drug. In the case of sunitinib, the maximum tolerated dose in children is $15 \mathrm{mg} / \mathrm{m}^{2}$, and in adults, $28 \mathrm{mg} / \mathrm{m}^{2(13)}$.

Reduced left ventricular ejection fraction was also evidenced in idarubicin therapy, occurring in $6 \%$ of cases $^{(20-21,30)}$. Idarubicin is an analogue of doxorubicin, which is equally effective and less cardiotoxic than other intravenous anthracyclines ${ }^{(36)}$. In the studies analyzed, the patients' ventricular function returned to normal a few months after chemotherapy was finished, without the development of permanent cardiac dysfunction ${ }^{(20)}$. The dose of idarubicin used ranged from $45 \mathrm{mg} / \mathrm{m}^{2}$ to $>400 \mathrm{mg} /$ $\mathrm{m}^{2}$, exceeding the toxic dose of the drug $\left(150-290 \mathrm{mg} / \mathrm{m}^{2}\right)^{(37)}$, which may have influenced the occurrence of adverse events. In the use of adequate dose, the study reports the absence of cardiotoxicity of the drug ${ }^{(30)}$.

Changes in electrocardiogram involved arrhythmias, conduction disorders, premature atrial contraction, bradycardia, T-wave alternans, QT interval changes, ST depression, QRS amplitude variation and atrial fibrillation. Transient and mild electrocardiographic changes were verified with idarubicin ${ }^{(30)}$. However, capecitabine can cause ventricular arrhythmias and atrial fibrillation, but it is less cardiotoxic than its 5-fluorouracil intravenous analog ${ }^{(38)}$. Tyrosine kinase inhibitors such as sunitinib, sorafenib, pazopanib, nilotinib and dasatinib also cause these events ${ }^{(27)}$.

In relation to sunitinib and/or sorafenib therapy, rhythm abnormalities, conduction disorders, QRS amplitude variations, ST depression and elevation, T-wave alternans and QT interval prolongation were observed, with emphasis on T-wave alternans ${ }^{(12)}$. In the treatment with pazopanib, a decrease in heart rate in a dose-dependent manner and change in the QT interval, besides transient dysfunctions such as sinus bradycardia, ectopic supraventricular rhythms, atrioventricular block and ST depression have been reported. Therefore, these medicinal products should be administered with caution in individuals with arrhythmia and sinus node dysfunction ${ }^{(31)}$. QT prolongation may also be evidenced in treatment with dasatinib and nilotinib, in addition to arrhythmias, conduction disorders and changes in the ST segment. Thus, cardiac monitoring during treatment with tyrosine kinase inhibitors is recommended because of the increased risk of occurrence of malignant arrhythmias ${ }^{(19,32)}$.

Ischemic heart diseases may occur with sunitinib, sorafenib, nilotinib, imatinib, capecitabine, tamoxifen and anastrozole, including angina, ischemia and myocardial infarction, leading to death ${ }^{(14,25,32)}$. Myocardial ischemia, chest pain and myocardial infarction occurred after treatment with imatinib in patients with sarcoma ${ }^{(16)}$. Myocardial infarction during monotherapy with sunitinib has been verified in patients with renal cell carcinoma or gastrointestinal stromal tumor ${ }^{(14,25)}$. Angina may be late in therapy with nilotinib, whereas, in association with capecitabine, it occurs between the second and fifth day of treatment ${ }^{(39-40)}$. These changes have also been reported in treatment with tamoxifen and anastrozole ${ }^{(29)}$.

Heart failure is one of the consequences associated with therapy with tyrosine kinase inhibitors ${ }^{(11)}$. In cases resulting from sunitinib, it occurs early and is reversible after stopping of therapy ${ }^{(13-14)}$. The main symptoms were fatigue and dyspnea, and the predictors of heart failure were coronary heart disease and hypertension ${ }^{(26)}$. The diagnosis of heart failure associated with sunitinib in patients with renal cell carcinoma may be difficult to recognize, since meaningful percentages of individuals with this tumor tend to develop dyspnea, peripheral edema, and fatigue ${ }^{(25)}$. Congestive heart failure may result from therapy with imatinib and is associated with significant fluid retention that may result in decompensated heart failure ${ }^{(41)}$. The change may also occur in treatment with lapatinib, however, the incidence of dysfunction is generally lower ${ }^{(42)}$.

Pericardial effusion is an adverse event of treatment with cabergoline and bromocriptine ${ }^{(33)}$, as well as treatment with sunitini $b^{(12)}$. These changes may be asymptomatic and reversible, being uncommon in these therapies ${ }^{(12,33)}$. Cardiogenic shock was observed in only one of the analyzed studies, occurring after 22 days of treatment with sunitinib, so that emergency intubation and advanced support were required. Such alteration may be associated, in addition to the cardiotoxic potential of the drug itself, to the previous treatment with doxorubicin $450 \mathrm{mg} /$ $\mathrm{m}^{2(24)}$. Changes in cardiac enzymes may occur during therapy with sunitinib and sorafenib, with an increase in troponin and creatine kinase $\mathrm{MB}^{(12,15)}$. The investigation of these enzymes is relevant to clinical practice in the treatment of neoplasias, since these alterations may be associated with myocardial damage ${ }^{(25)}$.

Peripheral vascular events were: changes in blood pressure and thromboembolic events. Tyrosine kinase inhibitors were most associated with changes in blood pressure ${ }^{(11,15,18,25-26,28,31)}$, indicating the need for follow-up during the use of these therapies due to the negative impact they can cause in health. Studies have shown that the incidence of hypertension in sunitinib therapy was $37 \%$, whereas sorafenib was $36 \%{ }^{(11,25-26)}$. Hypertension is the most frequent adverse event in the treatment of renal cell 
carcinoma and gastrointestinal stromal tumor by sunitinib, being proportional to the period of time of treatment ${ }^{(43)}$. Increases in systolic and peripheral diastolic blood pressure were reported in a study with telatinib, affecting $78 \%$ of individuals with advanced solid tumors after five weeks of treatment. Some cases require antihypertensive therapy with the use of thiazide diuretics, calcium channel blockers or angiotensin converting enzyme inhibitors ${ }^{(18)}$. Increased blood pressure was observed in therapy with pazopanib in two studies reaching the incidence of $28 \%(11,31)$. In one of the clinical trials, there was a significant increase in systolic $(+17.7$ $\mathrm{mmHg}$ ) and diastolic ( $+11.9 \mathrm{mmHg}$ ) pressures on the ninth day of treatment, returning to the initial patterns after 24 hours $^{(31)}$.

Thromboembolic events have been reported in the treatment of breast cancer with anastrozole or tamoxifen, occurring peripheral vascular disorder and arteriosclerosis ${ }^{(29)}$. On treatment with sunitinib or sorafenib, ischemic optic neuropathy and occlusion of the posterior tibial artery were observed. Telatinib was also associated with vascular dysfunction, causing arterial stiffness, contributing to hypertension, as well as decreased skeletal muscle capillary density and decreased blood flow to the skin. Deep venous thrombosis, peripheral artery occlusion, and pulmonary thromboembolism were observed in the treatment by the combination of melphalan, prednisone and thalidomide, occurring mainly during the first four months of use. The cumulative incidence of thromboembolism is $20 \%$ in the absence of prophylactic anticoagulant, which makes it necessary to adopt this medication to prevent thrombotic events ${ }^{(23)}$.

Adverse cardiovascular events require a lot of care by the health professionals, among them the nurse. Since the planning of the oral antineoplastic treatment scheme, attention should be paid to individualized therapies that consider the characteristics and limitations of each patient. Thus, the health professional should know the pharmacological doses recommended for each population and the criteria for discontinuation of therapy.
Before and during treatment, it is necessary to guide patients about cardiac monitoring in order to prevent and diagnose the dysfunctions arising from therapy early. Depending on the drug, adverse events may occur sometime after completion of therapy. Thus, follow-up of these patients should be continued as recommended in each treatment regimen ${ }^{(44)}$.

\section{Study limitations}

As a limitation of this study, it was found that some articles analyzed used limited samples, which compromised the evidences in relation to the results of the research. Therefore, it is important to carry out more studies on the subject to compare and expand the results, since some samples were not representative.

Contributions to the area of nursing, health and public policy

This study is important because it points out the need for better monitoring of patients undergoing oral antineoplastic therapy, since many adverse events can go unnoticed if there is no adequate follow-up, so that only the most serious and fatal events are identified. These findings reinforce the need for knowledge about the adversities resulting from treatment for resolute and integral assistance, especially by the nurse, who is commonly present and responsible for identifying and alerting irregularities, providing clarification to the individual and family, and organizing and supervising the care provided by the nursing team.

\section{CONCLUSION}

Oral antineoplastic therapy is associated with different adverse events, including cardiac and peripheral vascular events. The most frequent adverse cardiac events were the reduced left ventricular ejection fraction, myocardial infarction, changes in electrocardiogram, heart failure and angina, whereas peripheral vascular events were hypertension and thromboembolism.

\section{REFERENCES}

1. Silva FCM, Comarella L. Efeitos adversos associados à quimioterapia antineoplásica: levantamento realizado com pacientes de um hospital do estado do Paraná. Rev Uniandrade [Internet]. 2013 [cited 2016 Oct 12];14(3):263-77. Available from: http://www. uniandrade.br/revistauniandrade/index.php/revistauniandrade/article/view/82/63

2. Oliveira AT, Ana PAQ. Perfil de uso da terapia antineoplásica oral: a importância da orientação farmacêutica. Rev Bras Farm Hosp Serv Saúde [Internet]. 2012 [cited 2016 Oct 12];3(4):24-9. Available from: http://www.sbrafh.org.br/rbfhss/public/artigos/2012030420BR.pdf

3. Suspiro A, Prista J. Occupational exposure to anticancer drugs and adverse health effects. Rev Port Saúde Públ [Internet]. 2012[cited 2016 Oct 12];30(1):76-88. Available from: http://www.elsevier.pt/pt/revistas/revista-portuguesa-saude-publica-323/ $\mathrm{pdf} / \mathrm{S} 087090251200003 \mathrm{X} / \mathrm{S} 300 /$

4. Sayin OA, Ozpeker C, Schoenbrodt M, Oz F, Borgermann J, Gummert J, et al. Ventricular assist devices in patients with chemotherapyinduced cardiomyopathy: new modalities. Acta Cardiol[Internet]. 2015 [cited 2016 Oct 12];70(4):430-4. Available from: https:// www.ncbi.nlm.nih.gov/pubmed/26455245

5. Harbeck N, Ewer MS, Laurentiis M, Suter TM, Ewer SM. Cardiovascular complications of conventional and targeted adjuvant breast cancer therapy. Ann Oncol[Internet]. 2011 [cited 2016 Oct 12];22(6):1250-8. Available from: http://annonc.oxfordjournals.org/ content/22/6/1250.full.pdf + html

6. Gozzo TO, Souza SG, Moysés AMB, Carvalho RAO, Ferreira SMA. Knowledge of a nursing team about chemotherapy adverse effects. Cienc Cuid Saude [Internet]. 2015[cited 2016 Oct 12];14(2):1058-66. Available from: http://periodicos.uem.br/ojs/index. php/CiencCuidSaude/article/view/25040/pdf_364 
7. Florescu M, Cinteza M, Vinereanu D. Chemotherapy-induced Cardiotoxicity. Maedica[Internet]. 2013 [cited 2016 Oct 12];8(1):5967. Available from: https://www.ncbi.nlm.nih.gov/pmc/articles/PMC3749765/pdf/maed-08-59.pdf

8. Mendes KDS, Silveira RCCP, Galvão CM. [Integrative literature review: a research method to incorporate evidence in health care and nursing]. Texto Contexto Enferm [Internet]. 2008 [cited 2012 May 05];17(4):758-64. Available from: http://www.scielo.br/pdf/ tce/v17n4/18.pdf Portuguese.

9. Santos CMC, Pimenta CAM, Nobre MRC. A estratégia PICO para a construção da pergunta de pesquisa e busca de evidências. Rev Latino-Am Enfermagem[Internet]. 2007 [cited 2017 Sep 05];15(3):508-11. Available from: http://dx.doi.org/10.1590/ S0104-11692007000300023

10. Melnyk BM, Fineout-Overholt E. Making the case for evidence-based practice. In: Melnyk BM, Fineout-Overholt E. Evidence-based practice in nursing \& healthcare: a guide to best practice. Philadelphia: Lippincot Williams \& Wilkins; 2005.

11. Hall PS, Harshman LC, Srinivas S, Witteles RM. The frequency and severity of cardiovascular toxicity from targeted therapy in advanced renal cell carcinoma patients. JACC: Heart Fail[Internet]. 2013 [cited 2016 Oct 16];1(1):72-8. Available from: http:// www.sciencedirect.com/science/article/pii/S2213177912000030

12. Schmidinger M, Zielinski CC, VogI UM, Bojic A, Bojic M, Schukro C, et al. Cardiac toxicity of sunitinib and sorafenib in patients with metastatic renal cell carcinoma. J Clin Oncol [Internet]. 2008 [cited 2016 Oct 16];26(32):5204-12. Available from: http://jco. ascopubs.org/content/26/32/5204.full.pdf + html

13. DuBois SG, Shusterman S, Ingle AM, Ahern CH, Reid JM, Wu B, et al. Phase I and pharmacokinetic study of sunitinib in pediatric patients with refractory solid tumors: a children's oncology group study. Clin Cancer Res [Internet]. 2011 [cited 2016 Oct 16];17(15):5113-22. Available from: https://www.ncbi.nlm.nih.gov/pmc/articles/PMC3149978/pdf/nihms303199.pdf

14. Tassi R, Baldazzi V, Lapini A, Carini M, Mazzanti R. Hyperlipidemia and hypothyroidism among metastatic renal cell carcinoma patients taking sunitinib malate: related or unrelated adverse events? Clin Genitour Cancer[Internet]. 2015[cited 2016 Oct 16];13(2):e101-5. Available from: http://www.clinical-genitourinary-cancer.com/article/S1558-7673(14)00202-X/pdf

15. Motzer RJ, Hutson TE, Tomczak P, Michaelson MD, Bukowski RM, Rixie O, et al. Sunitinib versus interferon alfa in metastatic renal-cell carcinoma. N Engl J Med [Internet]. 2007 [cited 2016 Oct 16];356:115-24. Available from: http://www.nejm.org/doi/ pdf/10.1056/NEJMoa065044

16. Trent JC, Patel SS, Zhang J, Araujo DM, Plana J, Lenihan DJ, et al. Rare incidence of congestive heart failure in gastrointestinal stromal tumor and other sarcoma patients receiving Imatinib Mesylate (IM). Cancer [Internet]. 2010[cited 2016 Oct 16];116(1):18492. Available from: https:/www.ncbi.nlm.nih.gov/pmc/articles/PMC4306337/pdf/nihms149947.pdf

17. Blackwell KL, Burstein HJ, Storniolo AM, Rugo H, Sledge G, Koehler M, et al. Randomized study of lapatinib alone or in combination with trastuzumab in women with ErbB2-Positive, Trastuzumab-Refractory Metastatic Breast Cancer. J Clin Oncol [Internet]. 2010[cited 2016 Oct 16];28:1124-30. Available from: http://jco.ascopubs.org/content/28/7/1124.full.pdf + html

18. Steeghs N, Gelderblom H, Roodt J, Christensen O, Rajagopalan P, Hovens M. Hypertension and rarefaction during treatment with Telatinib, a small molecule angiogenesis inhibitor. Clin Cancer Res [Internet]. 2008[cited 2016 Oct 16];14(11):3470-7. Available from: http://clincancerres.aacrjournals.org/content/14/11/3470.full-text.pdf

19. Johnson FM, Agrawal S, Burris H, Rosen L, Dhillon N, Hong D, et al. Phase 1 pharmacokinetic and drug-interaction study of Dasatinib in patients with advanced solid tumors. Cancer [Internet]. 2010[cited 2016 Oct 16];116:1582-91. Available from: http:// onlinelibrary.wiley.com/doi/10.1002/cncr.24927/pdf

20. Lopez M, Contegiacomo A, Vici P, loio CD, Lauro L, Pagliarulo C, et al. A prospective randomized trial of Doxorubicin versus Idarubicin in the treatment of advanced breast cancer. Cancer [Internet]. 1989[cited 2016 Oct 16];64:2431-6. Available from: http://onlinelibrary.wiley.com/doi/10.1002/1097-0142(19891215)64:12\%3C2431::AID-CNCR2820641206\%3E3.0.CO;2-1/pdf

21. Pui C, Graaf SSN, Dow LW, Rodman JH, Evans WE, Alpert BS, et al. Phase I clinical trial of orally administered 4-Demethoxydaunorubicin (Idarubicin) with pharmacokinetic and in vitro drug sensitivity testing in children with refractory leukemia. Cancer Res[Internet]. 1988[cited 2016 Oct 16];48:5348-52. Available from: http://cancerres.aacrjournals.org/content/48/18/5348.full-text.pdf

22. Cutsem EV, Findlay M, Osterwalder B, Kocha W, Dalley D, Pazdur R, et al, Capecitabine, an oral fluoropyrimidine carbamate with substantial activity in advanced colorectal cancer: results of a randomized phase ii study. J Clin Oncol [Internet]. 2000 Mar [cited 2016 Oct 16]; 18(6): 1337-45. Available from: https://www.ncbi.nlm.nih.gov/pubmed/10715306

23. Palumbo A, Bertola A, Musto P, Caravita T, Callea V, Nunzi M et al. Oral Melphalan, Prednisone, and Thalidomide for newly diagnosed patients with myeloma. Cancer [Internet]. 2005[cited 2016 Oct 16];104:1428-33. Available from: http://onlinelibrary. wiley.com/doi/10.1002/cncr.21342/pdf

24. Telli ML, Witteles RM, Fisher GA, Srinivas S. Cardiotoxicity associated with the cancer therapeutic agent sunitinib malate. Ann Oncol[Internet]. 2008[cited 2016 Oct 16];19:1613-8. Available from: http://annonc.oxfordjournals.org/content/19/9/1613.full. $\mathrm{pdf}+\mathrm{html}$

25. Chu TF, Rupnick MA, Kerkela R, Dallabrida SM, Zurakowski D, Nguyen L, et al. Cardiotoxicity associated with the Tyrosine Kinase Inhibitor Sunitinib. Lancet [Internet]. 2007[cited 2016 Oct 16];370(9604):2011-9. Available from: https://www.ncbi.nlm.nih.gov/ pmc/articles/PMC2643085/pdf/nihms37146.pdf

26. Lorenzo G, Autorino R, Bruni G, Carteni G, Ricevuto E, Tudini M, et al. Cardiovascular toxicity following sunitinib therapy in 
metastatic renal cell carcinoma: a multicenter analysis. Ann Oncol[Internet]. 2009[cited 2016 Oct 16];20:1535-42. Available from: http://annonc.oxfordjournals.org/content/20/9/1535.full.pdf + html

27. Ghatalia P, Je Y, Kaymakcalan MD, Sonpavde G, Choueiri TK. QTc interval prolongation with vascular endothelial growth factor receptor tyrosine kinase inhibitors. British J Cancer [Internet]. 2015[cited 2016 Oct 16];112(2):296-305. Available from: https:// www.ncbi.nlm.nih.gov/pmc/articles/PMC4453446/pdf/bjc2014564a.pdf

28. Demetri GD, Oosterom ATV, Garrett CR, Blackstein ME, Shah MH, Verweij J. Efficacy and safety of sunitinib in patients with advanced gastrointestinal stromal tumour after failure of imatinib: a randomised controlled trial. Lancet [Internet]. 2006[cited 2016 Oct 16];368(9544):1329-38. Available from: http://www.thelancet.com/pdfs/journals/lancet/PIIS0140-6736(06)69446-4.pdf

29. The Arimidex, Tamoxifen, Alone or in combination Trialists' Group. Buzdar A, Howell A, Cuzick J, Wale C, Distler W, HoctinBoes G, et al. Comprehensive side-effect profile of anastrozole and tamoxifen as adjuvant treatment for early-stage breast cancer: long-term safety analysis of the ATAC trial. Lancet Oncol [Internet]. 2006[cited 2016 Oct 16];7:633-43. Available from: http:// www.thelancet.com/pdfs/journals/lanonc/PIIS1470-2045(06)70767-7.pdf

30. Martoni A, Pacciarini MA, Pannuti F. Activity of 4-Demethoxydaunorubicin by the Oral Route in Advanced Breast Cancer. Eur J Cancer Clin Oncol [Internet]. 1985 Jul [cited 2016 Oct 16]; 21(7): 803-6. Available from: https://www.ncbi.nlm.nih.gov/pubmed/3862582

31. Heath EI, Infante J, Lewis LD, Luu T, Stephenson J, Tan AR, et al. A randomized, double-blind, placebo-controlled study to evaluate the effect of repeated oral doses of pazopanib on cardiac conduction in patients with solid tumors. Cancer Chemother Pharmacol [Internet]. 2013 [cited 2016 Oct 16];71(3):565-73. Available from: https://www.ncbi.nlm.nih.gov/pmc/articles/PMC3899892/pdf/nihms-516601.pdf

32. Kim TD, Coutre P, Schwarz M, Grille P, Levitin M, Fateh-Moghadam S, et al. Clinical cardiac safety profile of nilotinib. Haematol[Internet]. 2012[cited 2016 Oct 16];97(6):883-9. Available from: https://www.ncbi.nlm.nih.gov/pmc/articles/PMC3366654/pdf/0970883.pdf

33. Elenkova A, Shabani R, Kalinov K, Zacharieva S. Increased prevalence of subclinical cardiac valve fibrosis in patients with prolactinomas on long-term bromocriptine and cabergoline treatment. European J Endocrinol[Internet]. 2012 [cited 2016 Oct 16];167(1):17-25. Available from: http://www.eje-online.org/content/167/1/17.full.pdf + html

34. Ezaz G, Long JB, Gross CP, Chen J. Risk prediction model for heart failure and cardiomyopathy after adjuvant trastuzumab therapy for breast cancer. J Am Heart Assoc [Internet]. 2014[cited 2016 Oct 16];3:e000472. Available from: http://jaha.ahajournals.org/ content/3/1/e000472.full.pdf + html

35. Saad ED, Hoff PM, Carnelós RP, Katz A, Novis YAS, Pietrocola M, et al. Critérios comuns de toxicidade do Instituto Nacional de Câncer dos Estados Unidos. Rev Bras Cancerol[Internet]. 2002 [cited 2016 Oct 16];48(1):63-96. Available from: http://www.inca. gov.br/rbc/n_48/v01/pdf/artigo6.pdf

36. Hohloch K, Zwick C, Ziepert M, Hasenclever D, Kaiser U, Engert A, et al. Significant dose Escalation of Idarubicin in the treatment of aggressive Non-Hodgkin Lymphoma leads to increased hematotoxicity without improvement in efficacy in comparison to standard CHOEP-14: 9-year follow up results of the CIVEP trial of the DSHNHL. Springerplus [Internet] 2014 [cited 2016 Oct 16];3:5. Available from: https://springerplus.springeropen.com/articles/10.1186/2193-1801-3-5

37. Anderlini P, Benjamin RS, Wong FC. Idarubicin cardiotoxicity: a retrospective study in acute myeloid leukemia and myelodysplasia. J Clin Oncol [Internet]. 1995 [cited 2016 Oct 16];13(11):2827-2834. Available from: http://jco.ascopubs.org/content/13/11/2827. abstract

38. Rosa GM, Gigli L, Tagliasacchi MI, Di lorio C, Carbone F, Nencioni A, et al. Update on cardiotoxicity of anti-cancer treatments. Eur J Clin Invest [Internet]. 2016[cited 2016 Oct 16];46 (3):264-84. Available from: http://onlinelibrary.wiley.com/doi/10.1111/ eci.12589/pdf

39. Van Cutsem E, Hoff PM, Blum JL, Abt M, Osterwalder B. Incidence of cardiotoxicity with the oral fluoropyrimidine capecitabine is typical of that reported with 5-fluorouracil. Ann Oncol [Internet]. 2002 [cited 2016 Oct 16];13:484-5. Available from: http:// annonc.oxfordjournals.org/content/13/3/484.full.pdf + html

40. Meyer CC, Calis KA, Burke LB, Walawander CA, Grasela TH. Symptomatic cardiotoxicity associated with 5-fluorouracil. Pharmacother[Internet]. 1997[cited 2016 Oct 16];17:729-36. Available from: http://onlinelibrary.wiley.com/doi/10.1002/j.1875-9114.1997. tb03748.x/epdf

41. Ewer MS, Ewer SM. Cardiotoxicity of anticancer treatments. Nature Rev Cardiol[Internet]. 2015[cited 2016 Oct 16];12:547-58. Available from: http://www.nature.com/nrcardio/journal/v12/n9/full/nrcardio.2015.65.html

42. Suter TM, Procter M, van Veldhuisen DJ, Muscholl M, Bergh J, Carlomagno C, et al. Trastuzumab-associated cardiac adverse effects in the herceptin adjuvant trial. J Clin Oncol [Internet]. 2007[cited 2016 Oct 16];25:3859-65. Available from: http://jco.ascopubs. org/content/25/25/3859.full.pdf + html

43. Ewer MS, Suter TM, Lenihan DJ, Niculescu L, Breazna A, Demetri GD, et al. Cardiovascular events among 1090 cancer patients treated with sunitinib, interferon, or placebo: a comprehensive adjudicated database analysis demonstrating clinically meaningful reversibility of cardiac events. European J Cancer [Internet]. 2014[cited 2016 Oct 16];50:2162-70. Available from: http://jco. ascopubs.org/content/25/25/3859.full.pdf + html

44. Kalil Filho R, Hajjar LA, Bacal F, Hoff PM, Diz M del P, Galas FRBG. I Diretriz Brasileira de Cardio-Oncologia da Sociedade Brasileira de Cardiologia. Arq Bras Cardiol[Internet]. 2011[cited 2016 Oct 26];96(2):01-52. Available from: http://dx.doi.org/10.1590/ S0066-782X2011000700001 